\section{REVISED CLASSIC}

\section{Eugene Wolf's Anatomy of the Eye and Orbit}

Including the Central Connections, Development, and Comparative Anatomy of the Visual Apparatus. Sixth edition revised by $R$. J. Last. Pp. vii +529 (465 illustrations). (H. K. Lewis: London, 1968.) 105s.

WHAT is the proper treatment for the standard textbooks of the past? They could be allowed to die quietly, perhaps to become monuments of the past and documents for the historian. They could be corrected and brought up to date where absolutely necessary, but otherwise still allowed to bear the character imposed by the author. Thirdly, they could be rewritten "in the modern idiom" and so be transformed into a virtually new text. There is something to be said in favour of each of these possibilities. Wolff's Anatomy of the Eye and Orbit has recently undergone the second of these treatments.

The concept of anatomy in 1968 has clearly changed very notably from that in 1933 , in which year this book was first produced as a text based on a series of lectures. The book is, therefore and inevitably, something of an anachronism. Anatomy of today includes far more of the microstructure within its framework than in 1933, and therein lies a difficulty, because, the more "micro" the approach, the more transient and changing do the "structures" seem to be. This means that the interpretation of function must enter more and more into the interpretation of structure, till eventually structure and function become one and the same, that is, in the arrangement and interplay of molecules. Is the distribution of enzymes "structure" or "function"? Many anatomists spend their lives trying to locate enzymes, believing them to be useful anatomical signposts. Yet, in this book on the anatomy of the eye, which has an important section on retinal structure, there is no mention of the localization or development of its highly characteristic enzymatic systems.

In the field of ophthalmology there has been, during the past twenty years, enormous progress in the understanding of retinal structure and function. and the two have really become inseparable. It is disappointing to find, therefore, that the part of the book on the retina has been rather unevenly, though extensively revised. For example, it still contains (and in italics, too, on page 139) the popular error that in the fovea centralis each cone is connected to only one ganglion cell. Polyak, whose work, incidentally, is insufficiently recognized in this book, made it quite clear in 1941, and others have since confirmed, that while there are probably ganglion cells (midgets) that receive information from only one cone, the cones probably disseminate their information to several bipolar cells (both midgets and flat-tops) and do this even in the central rod-free area.

There are curious omissions also in other chapters. In the description of the development of the eye, neither the peculiar distribution of the dividing cells of the retina nor the occurrence of the widespread degeneration of cells as part of the normal process of retinal development (in the frog at least) receive any mention, yet these are two aspects of the developmental process which are basic to the understanding of the manner in which the final structure is achieved.

The chapter on the comparative anatomy of the eye also suffers from the very restricted anatomical and histological approach. The recent advances in the investigation of the minute structure of photoreceptors in general might have been incorporated with advantage and it is odd to find no mention of the structure of the eye of Limulus, on which some of the most fundamental work on the physiology of vision has been performed.

In spite of these strictures, the field of vision is enormous, and the sixth edition of this well known anatomical text must still rank as an important source of useful and necessary information on the structural aspects of the eye and its associated tissues; and this is no mean achievement. The revision could have been more extensive with advantage, but the book would then be in danger of losing its initial character and purpose. As it is, the work provides an excellent and well-illustrated compendium of the sort of information thought to be necessary for the ophthalmologist, and it lays special emphasis on those topics that are of practical importance to him. There is plenty of life in the old dog yet and we trust that it will last for many more years, but let the editors remember that wolves thrive better on the living than on the dead.

E. N. Willmer

\section{AGEING AND PERFORMANCE}

\section{Human Aging and Behavior}

Recent Advances in Research and Theory. Edited by George A. Talland. Pp. xiii +322 . (Academic Press: New York and London, 1968.) 112s.

Thrs is an excellent, well produced book though by no means free of printing errors. It is about the effects of age on a number of physiological functions and various aspects of human performance. The late Dr Talland has succeeded in his aim of producing a textbook for advanced studies in this general area, because the contributors are competent in research and provide excellent surveys of the recent literature relevant to their particular interests. There is a little overlap between chapters but this is only to be expected, and even adds interest because students can discover how the same empirical data can provide material relevant to different theories about human behaviour and the effects of ageing.

In the first chapter Surwillo writes about the timing of behaviour in senescence and the role of the central nervous system. He provides a clear and up to date exposition of the relationships between electroencephalogram data and responses of different degrees of complexity, including reactions to the passage of time. Szafran then considers the effects of age on a variety of psychophysiological functions in a highly selected group of aircraft pilots. Like Surwillo's chapter, and indeed most chapters, Szafran's work requires close and repeated reading-not because of deficiencies in presentation but simply because the book is packed with information, much of it highly technical and occurring in the context of closely reasoned argument. Szafran's results are somewhat unexpected and, unless they can be accounted for by methodological and sampling considerations, seem to imply a need to reconsider some aspects of the psychology of ageing; for example, discrimination and choice in well established, long practised skills. In the third chapter Rabbitt deals with age and the use of structure in transmitted information, that is, the relative inefficiency and slowness of older people in situations involving uncertainty. This chapter is brief and lucid; Rabbitt spells out the positive analogy between the ageing human operator and aspects of computer functions, and makes useful suggestions about directions for future research. In chapter 4, Talland deals with age and the span of immediate recall. He describes a series of highly specialized experiments on the effects of ageing on short term memory (STM) under various conditions. Theoretical treatment is minimal in this chapter, although most authors try to provide the theoretical framework necessary to give point to their experimental investigations. Craik then writes about STM and the ageing process. He presents an excellent account of a connected series of experiments arising out of attempts to formulate an adequate theory. STM is not yielding easily to experimental psychological analysis and it will be interesting to see what sort of solution to the 